\title{
Rendimiento académico de la asignatura "Metodología de la investigación científica" en las diferentes carreras de la ESPOCH sede Morona Santiago
}

Rendimiento académico de la asignatura "Metodología de la investigación
científica" en las diferentes carreras de la ESPOCH sede Morona Santiago

Segundo Enrique Vaca Zambrano. ${ }^{1}$, Georing Octavio Zambrano Cardenas. ${ }^{2}$, Diego Iván Cajamarca Carrazco. ${ }^{3} \&$ Kathia Dayanara Loja Quichimbo. ${ }^{4}$

Recibido: 18-11-2020 / Revisado: 15-12-2021 /Aceptado: 09-01-2021/ Publicado: 05-02-2021

\begin{abstract}
.
DOI: https://doi.org/10.33262/concienciadigital.v4i1.1.1552
\end{abstract}

Introduction. Academic performance is a quantitative methodology that allows to measure the amount of information received by students after having completed an academic period, which must be at least $70 \%$ to promote to the next higher grade. Objective. Determine the academic performance of the subject Methodology of Scientific Research in the Polytechnic School of Chimborazo, Morona Santiago headquarters. Methodology: the research was developed considering the 64 students: 32 from the Environmental Engineering career, 17 from the Zootechnical Engineering career and 15 from the Mining career; For which the academic evaluation records of the first, second, third partial and final of the period between September 2018 - February 2019 were used, the results were analyzed with the unbalanced statistical model and comparison of means according to Tukey $(p<0.05)$. Results: The students of the Environmental engineering career in the first quarter reached an average of 7.16 / 8.00 points, being statistically different $(p<0.01)$ from the average reached by the students of the Zootechnics and Mines career. The academic performance of the group of students from the three careers analyzed in the second and third quarter was not significant ( $\mathrm{p}$ 0.05), while for the final exam the highest performance corresponds to the Environmental

\footnotetext{
${ }^{1}$ Escuela Superior Politécnica de Chimborazo Sede Morona Santiago. enrique.vaca@espoch.edu.ec, https://orcid.org/0000-0003-2011-3128

${ }^{2}$ Escuela Superior Politécnica de Chimborazo Sede Morona Santiago. georingzambrano@espoch.edu.ec, https://orcid.org/0000.0001-6975-8539

${ }^{3}$ Escuela Superior Politécnica de Chimborazo Sede Morona Santiago. diego.cajamarca@espoch.edu.ec, https://orcid.org/0000-0003-2011-3128

${ }^{4}$ Investigadora independiente, Morona Santiago. Katia.loja@ hotmail.com,
} 
Engineering career and the students of the career Zootechnics registered the lowest average.

Key words: Methodology, performance, motivation, intelligence and cognitive.

\section{Resumen.}

Introducción. El rendimiento académico es una metodología cuantitativa que permite medir la cantidad de información receptada por los educandos luego de haber cursado un periodo académico, el mismo que debe ser al menos el $70 \%$ para promover al curso inmediato superior. Objetivo. Determinar el rendimiento académico de la asignatura Metodología de la Investigación Científica en la Escuela Superior politécnica de Chimborazo sede Morona Santiago. Metodología: la investigación se desarrolló considerando los 64 alumnos: 32 de la carrera de Ingeniería Ambiental, 17 de la carrera de Ingeniería Zootecnia y 15 de la carrera de Minas; para lo cual se utilizaron las actas de evaluación académica del primero, segundo, tercer parcial y final del periodo comprendido entre septiembre 2018 - febrero 2019, los resultados fueron analizados con el modelo estadístico desbalanceado y comparación de medias según Tukey $(\mathrm{p}<0.05)$. Resultados: Los estudiantes de la carrera de ingeniería Ambiental en el primer parcial alcanzaron un promedio de 7.16/8.00 puntos, siendo diferente estadísticamente $(\mathrm{p}<0.01)$ del promedio alcanzado por los estudiantes de la carrera de Zootecnia y Minas. El rendimiento académico del grupo de estudiantes de las tres carreras analizadas en el segundo y tercer parcial no se registraron significancias ( $p>0.05)$, mientras que para el examen final el mayor rendimiento corresponde a la carrera de Ingeniería Ambiental y los estudiantes de la carrera de Zootecnia registraron el promedio más bajo.

Palabras claves: Metodología, rendimiento, motivación, inteligencia y cognitiva.

\section{Introducción.}

La academia en la práctica es un proceso metodológico que intervienen educadores y educandos sujetos a la legislación ecuatoriana en la cual deben cumplir disposiciones emanadas en estatutos, reglamentos y normas establecidas por las entidades de educación básica, media o superior con la finalidad de formar una sociedad competitiva en el desarrollo de la sociedad (Constitución de la Republica del Ecuador, 2008).

En el proceso enseñanza - aprendizaje, actúan dos actores sociales, en el cual el docente imparte el conocimiento (ciencia) y el estudiante aprende los contenidos establecidos en la malla micro curricular para su formación e integración en la sociedad ilustrada y de desarrollo gracias a la capacidad cognitiva del alumno que en su cerebro procesa mentalmente el conocimiento e implicaciones, cuya consecuencia permite el manejo de las autopercepciones, habilidades y esfuerzo (Edel, 2003). 
Desde cuando existió la formación académica, el profesor valoró el esfuerzo de sus educandos, reconoce la habilidad innata e impulsa la autoestima (Edel, 2003). De esta manera se identifica estudiantes con altos rendimientos que alcanzan el dominio del conocimiento, estudiantes que aceptan el fracaso, estudiantes que evitan el fracaso a través de fraudes conocidas como estrategias para promover al curso inmediato superior, sin considerar la responsabilidad que implica saber o no saber y su consecuencia en ejercicio de la vida profesional (Covington, 1984).

El éxito implica la autopercepción de habilidad de adquirir el conocimiento y el esfuerzo que no perjudica el reconocimiento del profesor al buen estudiante. Mientras que, la situación de fracaso involucra un sobre esfuerzo como compensación a su poca habilidad, que genera un sentimiento de humillación (Elden, 2003), esta particularidad de análisis genera duda a la capacidad y, para evitar utiliza mecanismos de escusa y manipulación al esfuerzo (Covington y Omelich, 1979), causa que ha permitido el deterioro del aprendizaje, esto implica una profecía de fracaso en su formación académica (Covington, 1984).

La motivación es uno de los aspectos decisivos en el proceso de formación profesional (Nicholls, 1989) se ha considerado fundamental en el aprendizaje (Duda, 2001) en los estudiantes, tiene un significado contextual para demostrar su capacidad cognitiva. Si no esta motivado, se crea una barrera de incertidumbre trayendo como consecuencia la deserción y falta de interés por sus estudios. Siempre será importante la motivación debe ser promovida en los actores sociales que se encuentran inmersos en el proceso de su formación profesional, de esto depende el éxito o fracaso (Duda, 2001). En muchas ocasiones se juzgan en vez de motivar, lo que hace que influya a la hora de adoptar una $\mathrm{u}$ otra orientación. Por otro lado, la motivación permite un ego, que se relaciona con el deseo de demostrar mayor capacidad de tener mejores rendimientos frente a los demás que implica el interés por aprender y progresar (Cecchini, González, Carmona y Contreras, 2004; Duda, 2001; Ferrer-Caja y Weiss, 2000; Gutiérrez y García-Ferriol, 2001).

La motivación permite obtener beneficios psicológicos gracias a una buena orientación, característica que permite contrarrestar al ego como se ha demostrado en diferentes documentados en las últimas décadas (Duda, 2001). En este sentido, se han destacado que la orientación motivacional es una tarea de motivación intrínseca (Cervelló, Escartí y Balagué, 2000; Duda, Chi, Newton, Walling y Catley, 1995; Kim, Williams y Gill, 2003).

Existen factores que influyen el rendimiento académico desde los docentes tales como: la pedagogía, la didáctica, el dominio de la asignatura, la metodología y técnicas de estudio, los valores humanos, estado psicológico, experiencia y vocación del docente, por parte del estudiante se considera a los siguientes factores: socioeconómica, política, religiosa, salud, cultura, estado psicológico y motivacional, sentimental a ello se suma la amplitud de los programas de estudio, la enseñanza personalizada, conceptos previos y, el nivel de 
pensamiento (Benítez, Giménez y Osicka, 2000), Jiménez (2000) hace referencia a que "se puede tener una buena capacidad intelectual y una buena aptitud, sin embargo no estar obteniendo un rendimiento adecuado", ante este problema, el rendimiento académico es un fenómeno multifactorial.

Basados en esta premisa la presente investigación analiza, el nivel de conocimientos demostrado en la metodología de la investigación científica (Rendimiento académico) de los estudiantes de las carreras de Ingeniería Ambiental, Zootecnia y Minas de la ESPOCH - sede Morona Santiago, obtenido de las actas de evaluación permanente (Jiménez, 2000), esta evaluación permite tomar acciones inmediatas encaminadas al mejoramiento de la calidad educativa en el nivel superior.

\section{Materiales y Métodos.}

Para realizar el análisis del rendimiento académico de la catedra "Metodología de la Investigación científica" se utilizó las actas académicas de 64 estudiantes distribuidos en las carreras de Ingeniería Ambiental, Zootecnia y Minas (32, 17 y 15 respectivamente), los cuales estuvieron matriculados y constan en las actas de la ESPOCH sede Morona Santiago en el periodo marzo - julio 2019; el docente que impartió esta asignatura a los tres paralelos utilizó una metodología uniforme de enseñanza aprendizaje con la finalidad de evitar variación.

El ambiente en el cual recibieron clases el grupo de estudiantes de cada carrera tiene un espacio adecuado, con suficiente ventilación, iluminación y libres ruidos extraños que afecten al proceso de enseñanza aprendizaje, la capacidad de las aulas albergan 40 alumnos por sala, aunque en algunas de ellas varían la densidad en función del número de estudiantes matriculados por carrera, generalmente son inferiores a las construidas técnicamente establecidas.

El rendimiento académico fue obtenido mediante: pruebas, tareas, deberes, lecciones, talleres y evaluaciones periódicas durante el semestre, fueron adaptados a un modelo lineal aditivo $\mathrm{Yij}=\mathrm{u}+\mathrm{Ti}$. + Eij, donde $\mathrm{Yij}$ : es el valor estimado del rendimiento académico obtenido de cada estudiantes por efecto de las carreras que están cursando, u: es el promedio general, $\mathrm{Tj}$, es el efecto de las carreras (Ingeniería Ambiental, Zootecnia y Minas) en las que imparte la catedra "Metodología de la investigación científica" y Eij: es el efecto del rendimiento individual cada uno de los alumnos de las carreras. Los resultados obtenidos de las actas fueron procesados y analizados con la varianza desbalanceada y la comparación de medias según la técnica de Tukey utilizando el paquete estadístico Excel e Infostat versión estudiantil 3.3.2.

\section{Resultados y Discusión.}

El rendimiento promedio de los estudiantes de la carrera de ingeniería Ambiental fue de 7.16 / 8.00 puntos que corresponden al primer parcial en la asignatura de Metodología de 
investigación científica, valor que difiere significativamente $(\mathrm{p}<0.01)$ del rendimiento promedio alcanzado por los estudiantes de las carreras de Zootecnia y Minas que registraron 6.59 y 6.00/8.00 puntos respectivamente, posiblemente esto puede deberse al dialecto utilizado en la asignatura de mantera técnica y general, o a su vez a que los estudiantes recién están incursionando en el área de la investigación científica y les falta dominio para poder desenvolverse adecuadamente en este ámbito científico.

En la educación publica auspiciado por el Estado a nivel básico y medio poco o nada han hecho para que los educandos se formen haciendo investigación, sino únicamente han transmitido lo que esta descubierto (Carobone, 2013). Esta particularidad a propiciado en los estudiantes que cursan los primeros niveles universitarios de las carreras de Zootecnia y Minas en la ESPOCH sede Morona Santiago, la falta de interés por el desarrollo del conocimiento científico, viéndose afectado su rendimiento académico, razón por la cual es inevitable la transformación del modelo educativo que responda a los interés de la sociedad en búsqueda de dar soluciones a los problemas que requiere un mundo en vías de desarrollo (Gómez et al. 2014)

El rendimiento promedio de los estudiantes de Metodología de la educación científica en el segundo parcial de las carreras de Ambiental, Zootecnia y Minas alcanzaron 8.09, 8.35 y 8.20/10.00 puntos, valores que no registran diferencias significativas ( $p>0.05$ ) por efecto del lenguaje que se utiliza en cada una de las carreras, determinándose que, los estudiantes se han adaptado al lenguaje universal de la asignatura en mención, únicamente notándose una ligera variación en los estudiantes de la carrera de ingeniería Ambiental puesto que su rendimiento ha sufrido un descenso del 89.50 al $80.90 \%$, en cambio los alumnos de las carreras restantes demuestran superioridad con relación a la primera evaluación.

La superioridad del rendimiento académico de los estudiantes de Zootecnia y Minas posiblemente se deba a la motivación constante del educando de la asignatura Metodología de la Investigación científica a sus alumnos (Federación de enseñanza CC. OO., 2009) en donde pertinazmente se aplaca la generación del conocimiento a través de la investigación científica que permite solucionar problemas de la vida cotidiana, además de buscar la verdad absoluta con interés competitivo (Díamond, 2012)

Tabla 1: Rendimiento académico en la asignatura de Metodología de la investigación científica en el primero, segundo, tercer parcial y examen principal

\begin{tabular}{lrlrrrrrr}
\hline & \multicolumn{9}{c}{ Carreras } & & \\
\cline { 2 - 5 } Evaluaciones & Ambiental & \multicolumn{2}{c}{ Zootecnia } & \multicolumn{2}{c}{ Minas } & & E.E. & Prob. \\
\hline 1er parcial & 7,16 & $\mathrm{a}$ & 6,59 & $\mathrm{~b}$ & 6,00 & $\mathrm{c}$ & 0,17 & 0,00 \\
2do parcial & 8,09 & $\mathrm{a}$ & 8,35 & $\mathrm{a}$ & 8,20 & $\mathrm{a}$ & 0,23 & 0,81 \\
3er parcial & 8,78 & $\mathrm{a}$ & 8,59 & $\mathrm{a}$ & 9,93 & $\mathrm{a}$ & 0,32 & 0,08 \\
Principal & 11,19 & $\mathrm{a}$ & 6,90 & $\mathrm{~b}$ & 10,87 & $\mathrm{a}$ & 0,46 & 0,00 \\
\hline
\end{tabular}


Letras iguales horizontalmente no difiere significativamente $(\mathrm{p}>0.05)$.

E.E. Error experimental.

$\mathrm{Al}$ analizar el rendimiento académico del tercer parcial de los estudiantes de la asignatura Metodología de la Investigación científica en las carreras de ingeniería Ambiental, Zootecnia y Minas registraron notas de 8.78, 8.59 y 9.93 / 10 puntos, valores entre los cuales no se encuentran diferencias relevantes ( $p>0.05$ ), únicamente se observa un ligero incremento en el promedio del rendimiento académico con relación al segundo parcial en los estudiantes de la carrera de Minas (Tabla 1).

Generalmente el mejoramiento del rendimiento académico se debe a múltiples factores tales como como el gusto por la asignatura, la claridad de la explicación y la motivación permanente que propician la atención del educando además del interés de los estudiantes por las clases (Hernández et al, 2012) que permite registrar apuntes de refuerzo, buscar nueva bibliografía de su interés, participar activamente en clases con preguntas lógicas y evitando distraer la mente en otras situaciones. De la misma manera el mejoramiento del rendimiento académico se puede atribuir a la inteligencia estática (teoría psicométrica) y la dinámica (teoría cognitiva) (Villanueva, 2014). Otro de los aspectos fundamentales que afecta el rendimiento académico es la perspectiva biológica que tiene el estudiante para adaptarse. De otra manera Hebb aduce que la capacidad innata del cerebro capta la información del medio, almacena y utiliza en un momento dado (Monteros, 2006).

En relación con la evaluación final, como establece el reglamento de régimen académico de la ESPOCH en el artículo 70 literal b, es sobre doce (12) puntos; los estudiantes de las carreras de ingeniería Ambiental y Minas alcanzaron promedios de 11.19 y 10.80 / 12 puntos valore que difieren significativamente de los estudiantes de la carrera de Zootecnia los mismo que alcanzaron 6.90, siendo inferior al $70 \%$ mínimo para ser promovidos al curso inmediato superior. Esto posiblemente se deba a que en esta carrera las asignaturas son eminentemente practicas y poca teoría, lo que les hace complicado utilizar la teoría psicométrica (memorística) siendo en este campo necesario disponer de mayores horas para desarrollar actividades prácticas y obtener el conocimiento haciendo y/o investigando para lo cual se requiere un conjunto de etapas de la investigación científica (Ramos, 2008).

\section{Conclusiones.}

- Luego de un profundo análisis del rendimiento académico de los alumnos de la carrera de ingeniería ambiental fue superior a los estudiantes de las carreras de Ingeniería zootécnica y minas.

- Las carreras de, Ingeniería ambiental, zootecnia y minas se consideraron homogéneas estadísticamente en el segundo y tercer parcial. 
- Los estudiantes de la carrera de ingeniería ambiental y minas, luego de la evaluación final, se determinó que tienen mejores promedios que los estudiantes de zootecnia.

\section{Referencias Bibliográficas}

Benítez, M; Giménez, M. y Osicka, R. 2000. Las asignaturas pendientes y el rendimiento académico: ¿existe alguna relación? En red. Recuperado en:

http//fai.unne.edu.ar/links/LAS\%2...20EL\%20RENDIMIENTO\%20ACADEMICO.htm

Carobene, M, G. 2013. El conflicto de interés en la investigación científica. Rev Argent Microbiol Elsevie Doyma. 2013; 45(3) pp.145-146.

Cecchini, J. A., González, C., Carmona, A. M. y Contreras, O. (2004). Relaciones entre clima motivacional, la orientación de meta, la motivación intrínseca, la autoconfianza, la ansiedad y el estado de ánimo en jóvenes deportistas. Psicothema, 16, 104-109.

Cervelló, E., Escartí, A. y Balagué, G. (2000). Relaciones entre la orientación de meta disposicional y la satisfacción con los resultados deportivos, las creencias sobre las causas de éxito en deporte y la diversión con la práctica deportiva. Revista de Psicología del Deporte, 10, 2-16

Covington, M.; Omelich, C. 1979. ¿Are causal attributions causal?: A path analysis of the cognitive model of achievement motivation. Journal of Personality and Social Psychology, 37, pp. 1487-1504.

Covington, M. 1984. The motive for self-worth. En R. Ames y C. Ames (Eds.). Research on Motivation in Education. Student Motivation. Vol.I. New York: Academic Press.

Diamond. 2012. La motivación del científico. (en línea) consultado (19/07/2020) < https://www.gaussianos.com/la-motivacion-delcientifico/\#: :text=Cualquiera\%20que\%20afirme\%20que\%20los,habla\%20o\%20 miente\%20con\%20descaro.>

Duda, J. L. (2001). Achievement goal research in sport: Pushing the boundaries and clarifying some misunderstandings. En G. C. Roberts (Ed.), Advances in motivation in sport and exercise (pp.129-182). Champaign, IL.: Human Kinetics.

Duda, J. L., Chi, L., Newton, M. L., Walling, M. D. y Catley, D. (1995). Task and ego orientation and intrinsic motivation in sport. International Journal Sport Psychology, 26, 40-63 
Edel, R. 2003. El rendimiento académico: concepto, investigación y desarrollo. Revista Iberoamericana sobre Calidad, Eficacia y Cambio en Educación, vol. 1, núm. 2, julio-diciembre, p. 16.

Federación de Enseñanza CC. OO. 2009. Temas para la Educación - interés por el estudio en los adolescentes. Revista digital para profesionales de la enseñanza. (en línea) <https://www.feandalucia.ccoo.es/docu/p5sd5539.pdf> consultada (05/07/2020).

Ferrer-Caja, E. y Weiss, M. R. (2000). Predictors of intrinsic motivation among adolescent students in physical education. Research Quarterly for Exercise and Sport, 71, 267-279.

Gómez, J; Dieguez, D. y Gomez, D. 2014. Motivando el interés por la investigación científica en estudiantes de educación media superior. Congreso Iberoamericano de Ciencia, Tecnología, Innovación y Educación.

Gutiérrez, M. y García-Ferriol, A. (2001). El entorno escolar-familiar y la práctica deportiva en la adolescencia: una aproximación empírica. En J. Dosil (Ed.), Psicología y deporte de iniciación (pp. 35-55). Ourense: Gersam.

Hernández, C., Rodríguez, N. y Vargas, A. 2012. Los hábitos de estudio y motivación para el aprendizaje de los alumnos en tres carreras de ingeniería. Revista de la educación superior ISSN: 0185-2760 Vol. XLI (3), No. 163. Pp. 67 - 87.

(http://www.scielo.org.mx/pdf/resu/v41n163/v41n163a3.pdf)

Jiménez, M. 2000. Competencia social: intervención preventiva en la escuela. Infancia y Sociedad. 24, pp. 21-48.

Kim, B. J., Williams, L. y Gill, D. L. (2003). A cross-cultural study of achievement orientation and intrinsic motivation in young USA and Korean athletes. International Journal of Sport Psychology, 34, 168-184.

Monteros, J. 2006. Génesis de la teoría de las inteligencias múltiples. Revista Iberoamericana de Educación, 39, 1 - 3. Recuperado el 11 de mayo del 2014. http://www.rieoei.org/deloslectores/1263Monteros.pdf

Nicholls, J. (1989). The competitive ethos and democratic education. Cambridge, MA: Harvard University Press.

Ramos Chagoya Ena. 2008, julio 1). Métodos y técnicas de investigación. Recuperado de https://www.gestiopolis.com/metodos-y-tecnicas-de-investigacion/

RRAIG. 2014. Reglamento de Régimen académico de grado. ESPOCH. Editorial Multicolor. Riobamba - Ecuador. 
Villanueva, G. 2014. Las inteligencias múltiples por Howar Gadner Unidad propuestas para cambios metodológicos. Facultad de Educación - UNIR.

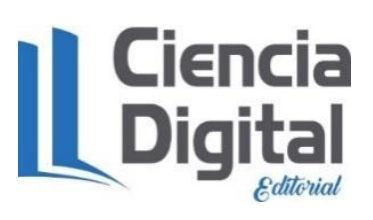




\section{PARA CITAR EL ARTÍCULO INDEXADO.}

Vaca Zambrano, S. E., Zambrano Cardenas, G. O., Cajamarca Carrazco, D. I., \& Loja Quichimbo, K. D. (2021). Rendimiento académico de la asignatura "Metodología de la investigación científica" en las diferentes carreras de la ESPOCH sede Morona Santiago. ConcienciaDigital, $4(1.1)$, 166-175. https://doi.org/10.33262/concienciadigital.v4i1.1.1552

\section{Liencia}

El artículo que se publica es de exclusiva responsabilidad de los autores y no necesariamente reflejan el pensamiento de la Revista Conciencia Digital.

El artículo queda en propiedad de la revista y, por tanto, su publicación parcial y/o total en otro medio tiene que ser autorizado por el director de la Revista Conciencia Digital.

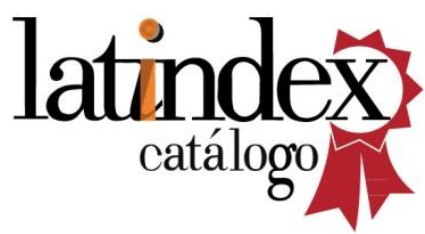

